\title{
A photometric study of the Bright Cloud B in Sagittarius
}

\section{1061 new variable stars $^{\star, \star \star, \star \star \star}$}

\author{
A. Terzan ${ }^{1}$, A. Bernard ${ }^{1}$, and J. Guibert ${ }^{2,3}$ \\ 1 Observatoire Astronomique de Lyon (CRAL), F-69561 Saint Genis Laval Cedex, France \\ 2 Centre d'Analyse des Images CNRS/INSU/MAMA, Observatoire de Paris, France \\ 3 Observatoire de Paris (DEMIRM)/URA 336 du CNRS, 77 Avenue Denfert-Rochereau, F-75014 Paris, France
}

Received August 8; accepted October 18, 1996

\begin{abstract}
We present a catalogue of positions and photographic $R$ magnitudes at observed maximum and minimum brightness for 1061 variable stars newly detected in the direction of the Bright Cloud B in Sagittarius. The limiting $R$ magnitude of the survey is about 18 . Most of these stars are long period variables. Taking into account the previously published lists (Terzan et al. 1982, 1988, 1991), the total number of new variable objects discovered in the surveyed 100 square degree field amounts to 4430 .
\end{abstract}

Key words: the Galaxy: structure; center - stars: variables; AGN, post-AGN

\section{Introduction}

A systematic photographic survey initiated in 1959 by A. Terzan (A. T.) in the direction of the Bright Cloud B in Sagittarius has been going on since 1976 at the ESO $1 \mathrm{~m}$ Schmidt telescope (Chile). A large number of peculiar objects have already been recognized, e.g. variable stars, galactic open clusters, galactic globular clusters, diffuse objects, planetary nebulae and proper motion stars (Terzan 1990 and references therein; Terzan \& Gosset 1991; Acker et al. 1992; Cuisinier et al. 1993, 1994).

Send offprint requests to: A. Terzan

* Plate scanning done with MAMA. MAMA is developed and operated by INSU (Institut National des Sciences de l'Univers).

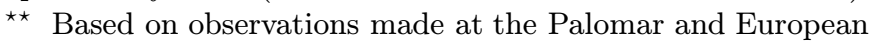
Southern Observatories.

$\star \star \star$ The whole of Table 3 , as well as the full catalogue of 4430 variables are only available in electronic form at CDS via anonymous ftp cdsarc.u-strasbg.fr (130.79.128.5) or via http://cdsweb.u-strasbg.fr/Abstract.html

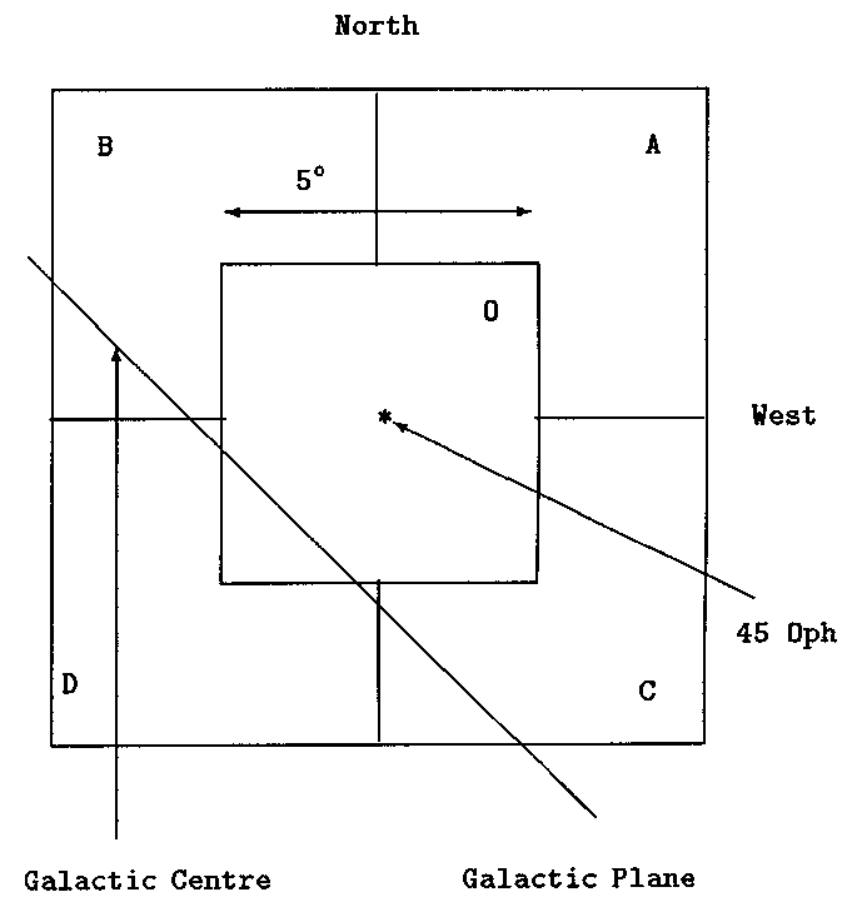

Fig. 1. Location of the $10^{\circ} \times 10^{\circ}$ area covered by fields $\mathrm{A}, \mathrm{B}$, $\mathrm{C}$ and D. Coordinates of star $45 \mathrm{Oph}$ can be found in the text

The $10^{\circ} \times 10^{\circ}$ programme field is centred on the star 45 Oph $\left(\alpha=17 \mathrm{~h} 27 \mathrm{~m} 21.2 \mathrm{~s} ; \delta=-29^{\circ} 52^{\prime} 01^{\prime \prime}\right.$, equinox 2000.0).

We present now the results of the search for long period variable stars in the last part of the programme field. The investigated region is a $5.5^{\circ} \times 5.5^{\circ}$ area, situated southwest the star $45 \mathrm{Oph}$, and defined by: $17 \mathrm{~h} 04 \mathrm{~m} \lesssim \alpha \lesssim$ $17 \mathrm{~h} 27 \mathrm{~m}$ and $-35^{\circ} 05^{\prime} \lesssim \delta \lesssim-29^{\circ} 52^{\prime}$. It corresponds to part $\mathrm{C}$ of the programme field (see Fig. 1, Table 1, and Fig. 2). A catalogue of positions and photographic $R$ magnitudes at observed maximum and minimum brightness is given for 1061 newly detected variables. The limiting 


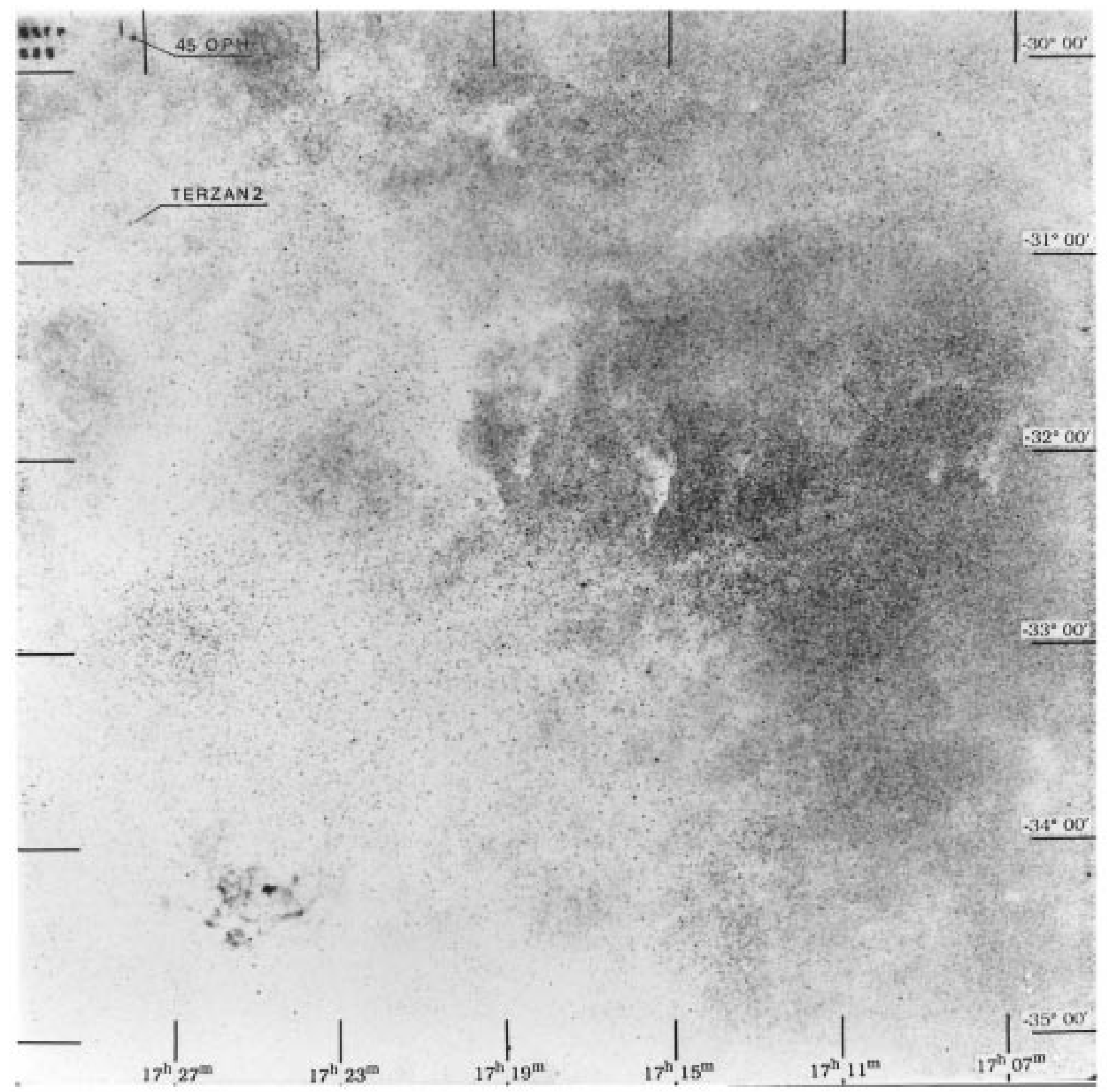

Fig. 2. Chart of Field C, south-west the star $45 \mathrm{Oph}$

magnitude of the detection is about 18. If we take into account the 3369 similar objects previoulsy discovered by Terzan et al. $(1982,1988,1991)$, the total number of new variable stars discovered in our 100 square degree field amounts to 4430. The global catalogue will be available in electronic form at CDS via anonymous ftp cdsarc.ustrasbg.fr or 130.79 .128 .5

\section{Observations and data reduction}

Our programme is based on photographic observations made in 1968 (june and july) at the $48^{\prime \prime}$ Schmidt telescope

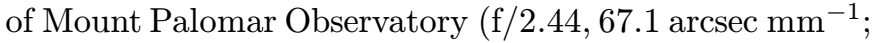
$B, V, R, 10^{\prime \prime} \times 10^{\prime \prime}$ plates) by A.T. continued, after 1976 , at the $1 \mathrm{~m}$ Schmidt telescope (f/3, $67.5 \operatorname{arcsec} \mathrm{mm}^{-1} ; U$, $B, V, R, 12^{\prime \prime} \times 12^{\prime \prime}$ plates) of ESO (Chile) by local staff observers.

For the present survey thirteen $R$ photographic plates have been retained for their photometric quality, at 
Table 1. Location of the investigated areas and number of detected variable stars

\begin{tabular}{ccccc}
\hline Field & Limits (Eq. 2000) & known variables (a) & detected variables (b) & new variables (c) \\
\hline A & $\begin{array}{l}17 \mathrm{~h} 04 \mathrm{~m} \lesssim \text { alpha } \lesssim 17 \mathrm{~h} 27 \mathrm{~m} \\
-29^{\circ} 52^{\prime} \lesssim \text { delta } \lesssim-24^{\circ} 42^{\prime}\end{array}$ & 97 & 1235 & $1178(\mathrm{~d}, \mathrm{e})$ \\
\hline $\mathrm{B}$ & $\begin{array}{l}17 \mathrm{~h} 27 \mathrm{~m} \lesssim \text { alpha } \lesssim 17 \mathrm{~h} 52 \mathrm{~m} \\
-29^{\circ} 52^{\prime} \lesssim \text { delta } \lesssim-24^{\circ} 42^{\prime}\end{array}$ & 56 & 1383 & $1383(\mathrm{~d}, \mathrm{f})$ \\
\hline $\mathrm{C}$ & $\begin{array}{l}17 \mathrm{~h} 04 \mathrm{~m} \lesssim \text { alpha } \lesssim 17 \mathrm{~h} 27 \mathrm{~m} \\
-35^{\circ} 05^{\prime} \lesssim \text { delta } \lesssim-29^{\circ} 52^{\prime}\end{array}$ & 46 & 1204 & $1061(\mathrm{~g})+130(\mathrm{~d})$ \\
\hline $\mathrm{D}$ & $\begin{array}{l}17 \mathrm{~h} 27 \mathrm{~m} \lesssim \text { alpha } \lesssim 17 \mathrm{~h} 52 \mathrm{~m} \\
-35^{\circ} 05^{\prime} \lesssim \text { delta } \lesssim-29^{\circ} 52^{\prime}\end{array}$ & 53 & 686 & $678(\mathrm{~d}, \mathrm{e})$ \\
\hline Total & & 252 & 4508 & 4430 \\
\hline
\end{tabular}

(a) GCVS (Kholopov et al. 1985, 1987);

(b) in the course of our programme;

(c) some low amplitude and/or blue objects of the Kukarkin's catalogue escaped the blink detection;

(d) Terzan et al. (1982);

(e) Terzan \& Ounnas (1988);

(f) Terzan \& Gosset (1991);

(g) this paper.

Table 2. Characteristics of the plates

\begin{tabular}{rcccllll}
\hline & Plate number & Date & Emulsion & Filter & Exposure & Seeing & Observatory \\
\hline 1 & 3809 & $1968-06-29$ & 103a-E & RG1 & $20 \mathrm{mn}$ & $2^{\prime \prime}$ & Palomar \\
2 & 3818 & $1968-06-30$ & 103a-E & RG1 & $20 \mathrm{mn}$ & $1^{\prime \prime}$ & Palomar \\
3 & 3879 & $1968-07-26$ & $103 \mathrm{a}-\mathrm{E}$ & RG1 & $20 \mathrm{mn}$ & $2^{\prime \prime}$ & Palomar \\
4 & 3899 & $1968-07-29$ & $103 \mathrm{a}-\mathrm{E}$ & $\mathrm{RG} 1$ & $20 \mathrm{mn}$ & $2^{\prime \prime}$ & Palomar \\
5 & 4810 & $1982-05-20$ & $098-04$ & RG630 & $30 \mathrm{mn}$ & $2^{\prime \prime}$ & ESO \\
6 & 7608 & $1988-10-02$ & $098-04$ & RG630 & $30 \mathrm{mn}$ & $1^{\prime \prime}$ & ESO \\
7 & 8005 & $1989-04-12$ & $098-04$ & RG630 & $30 \mathrm{mn}$ & $1.2^{\prime \prime}$ & ESO \\
8 & 8030 & $1989-05-09$ & $098-04$ & RG630 & $30 \mathrm{mn}$ & $1.5^{\prime \prime}$ & ESO \\
9 & 8081 & $1989-06-06$ & $098-04$ & RG630 & $30 \mathrm{mn}$ & $1.5^{\prime \prime}$ & ESO \\
10 & 9227 & $1991-04-19$ & $098-04$ & RG630 & $30 \mathrm{mn}$ & $1.5^{\prime \prime}$ & ESO \\
11 & 9234 & $1991-04-21$ & $098-04$ & RG630 & $30 \mathrm{mn}$ & $1^{\prime \prime}$ & ESO \\
12 & 9354 & $1991-07-10$ & $098-04$ & RG630 & $30 \mathrm{mn}$ & $1.5^{\prime \prime}$ & ESO \\
13 & 10636 & $1993-06-20$ & $098-04$ & RG630 & $30 \mathrm{mn}$ & $1.5^{\prime \prime}$ & ESO \\
\hline
\end{tabular}

observational time intervals suitable for the detection of long-period variable stars. The characteristics of the plates (plate number, date of the observation, emulsion, filter, exposure, and seeing conditions) are given in Table 2 . The comparison of photographic plates was done by one of us (A. T.) using the blink microscope of the Observatoire de Lyon, an instrument allowing stellar variations as small as 0.2 magnitude to be estimated on plates obtained in good seeing conditions ( $\lesssim 1$ arcsec) (Terzan et al. 1978). We found 1061 new variable stars in field $\mathrm{C}$, in addition to the 130 variables already detected in the area common to fields O and C (Terzan et al. 1982). Magnitudes have been estimated by scaling the photographic densities of variables to a sequence of densities corresponding to standard stars with $R$ magnitudes between 9 and
17.5 (Terzan \& Bernard 1981). Under these conditions, the uncertainty on $R$ is about 0.3 magnitude in the range $9 \lesssim R \lesssim 14$, and then increases up to 0.5 as $R$ approaches 18 , the magnitude limit of the estimations. The determination of the rectangular coordinates $X$ and $Y$ of the stars was made partly at ESO Garching with the microdensitometer S 3000- Optronics, partly with MAMA (Machine Automatique à Mesurer pour l'Astronomie). The astrometric reductions were performed using the PPM catalogue (Röser et al. 1991, 1993), with an internal accuracy of $\approx 0.2 \operatorname{arcsec}$ (see e.g. Robichon et al. 1996). 
Table 3. The first 16 new variable stars discovered in Field $\mathrm{C}$

\begin{tabular}{|c|c|c|c|c|c|c|c|c|c|c|}
\hline star number & $\alpha(2000)$ & $\delta(2000)$ & $l^{\mathrm{II}}$ & $b^{\mathrm{II}}$ & $R[\mathrm{Max}]$ & $E$ & $R[\mathrm{Min}]$ & $E$ & $R[\mathrm{Min}]-R[\mathrm{Max}]$ & Remarks \\
\hline 3380 & 170439.21 & -340502.6 & 350.8 & +04.3 & 11.9 & 13 & 18.0 & 7 & 6.1 & \\
\hline 3381 & 170444.01 & -334747.1 & 351.1 & +04.5 & 15.8 & 7 & $\gtrsim 18$ & 13 & & \\
\hline 3382 & 170448.71 & -333432.5 & 351.3 & +04.6 & 11.0 & 8 & 15.3 & 13 & 4.3 & \\
\hline 3383 & 170449.05 & -341423.1 & 350.7 & +04.2 & 11.0 & 8 & 15.8 & 13 & 4.8 & \\
\hline 3384 & 170455.37 & -330606.3 & 351.7 & +04.9 & 14.2 & 13 & 18.0 & 6 & 3.8 & \\
\hline 3385 & 170501.04 & -325914.7 & 351.8 & +04.9 & 14.2 & 8 & 17.6 & 6 & 3.4 & \\
\hline 3386 & 170504.96 & -331239.6 & 351.6 & +04.8 & 13.0 & 6 & 18.0 & 7 & 5.0 & \\
\hline 3387 & $\begin{array}{lll}17 & 05 & 07.97\end{array}$ & -312841.4 & 353.0 & +05.8 & 10.2 & 8 & 15.8 & 12 & 5.6 & \\
\hline 3388 & $\begin{array}{lll}17 & 05 & 17.04\end{array}$ & -330526.6 & 351.7 & +04.8 & 13.0 & 7 & 17.6 & 8 & 4.6 & \\
\hline 3389 & $1705 \quad 17.16$ & $-34 \quad 1223.7$ & 350.8 & +04.2 & 14.2 & 12 & 16.6 & 11 & 2.4 & \\
\hline 3390 & $\begin{array}{lll}17 & 05 & 19.92\end{array}$ & -321107.2 & 352.4 & +05.4 & 13.0 & 8 & 16.6 & 6 & 3.6 & \\
\hline 3391 & 170521.00 & -332636.0 & 351.4 & +04.6 & 14.2 & 6 & 18.0 & 7 & 3.8 & \\
\hline 3392 & 170521.43 & -332455.3 & 351.5 & +04.6 & 11.0 & 10 & 15.8 & 7 & 4.8 & \\
\hline 3393 & 170523.40 & -340304.4 & 351.0 & +04.2 & 15.0 & 8 & 18.0 & 11 & 3.0 & \\
\hline 3394 & 170523.87 & -302800.2 & 353.8 & +06.4 & 10.2 & 7 & 14.2 & 5 & 4.0 & \\
\hline 3395 & 170525.83 & $-32 \quad 1553.4$ & 352.4 & +05.3 & 15.8 & 6 & $\gtrsim 18$ & 7 & & \\
\hline
\end{tabular}

The whole of Table 3, as well as the full catalogue of the 4430 variables discovered in the $10^{\circ} \times 10^{\circ}$ programme field are available in electronic form at CDS via anonymous ftp cdsarc.u-strasbg.fr or 130.79.128.5

\section{The catalogue}

Astrometric and photometric results for the 1061 variables are catalogued in a table which is available in electronic form at CDS, and part of which is displayed for illustration as Table 3 in the printed version of this paper. This table also includes 13 variables belonging to the GCVS, but for which we provide identification charts as well as more accurate positions and Red magnitudes at observed maximum and minimum. The content of the successive columns is as follows:

Column 1: Star number. The numbering starts at 3380 for continuation of a previous list of already published variable stars (Terzan et al. 1982, 1988, 1991). The stars are classified according to increasing right ascension.

Columns 2 and 3: Equatorial coordinates referred to the equinox 2000.0.

Columns 4 and 5: Galactic coordinates $l^{\mathrm{II}}$ and $b^{\mathrm{II}}$.

Columns 6 and 8: $R[\mathrm{Max}]$ and $R[\mathrm{Min}]$, the $R$ magnitudes of the star at the observed maximum and minimum brightness.

Columns 7 and 9: E: Identification number of the plate corresponding to the epoch of observation of $R[\operatorname{Max}]$ and $R$ [Min] (Table 2).

Column 10: $A$ : Observed amplitude, $R[\mathrm{Min}]-R[\mathrm{Max}]$.

Column 11: Identification of the star in the GCVS catalogue (Kholopov et al. 1985, 1987). For IRxxx stars, see Terzan (1965) and references therein.

Finding charts for all the stars (from $R$ plates) are grouped on plates 1 to 17 . North is up and east is to the left, and scales are identical for all charts.

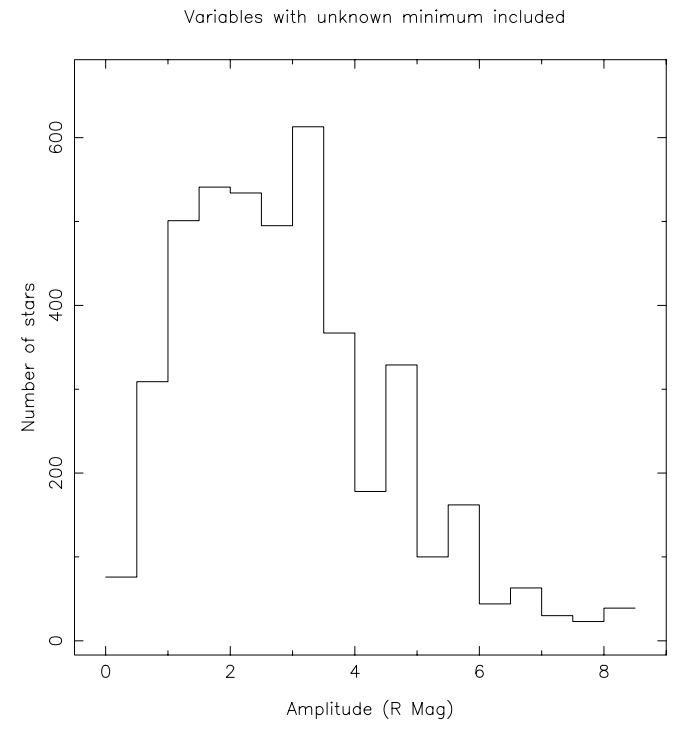

Fig. 3. Histogram of the observed amplitudes $A$, binned in steps of 0.5 magnitude, for the 2432 variables with definite $R$ amplitude

\section{Discussion}

An histogram of the number $N$ of variable stars as a function of the binned observed amplitude $A$, in steps of 0.5 magnitude, and for all stars with definite amplitude is represented in Fig. 3. For 50\% of the stars, the amplitudes range between 1.0 and 3.0, with a maximum for $1.5 \lesssim A \lesssim 2.0$. Ascribing magnitude 19 at brightness minimum to the stars with $R[\mathrm{Min}] \gtrsim 18$, one can build the corresponding histogram for almost all the catalogued 


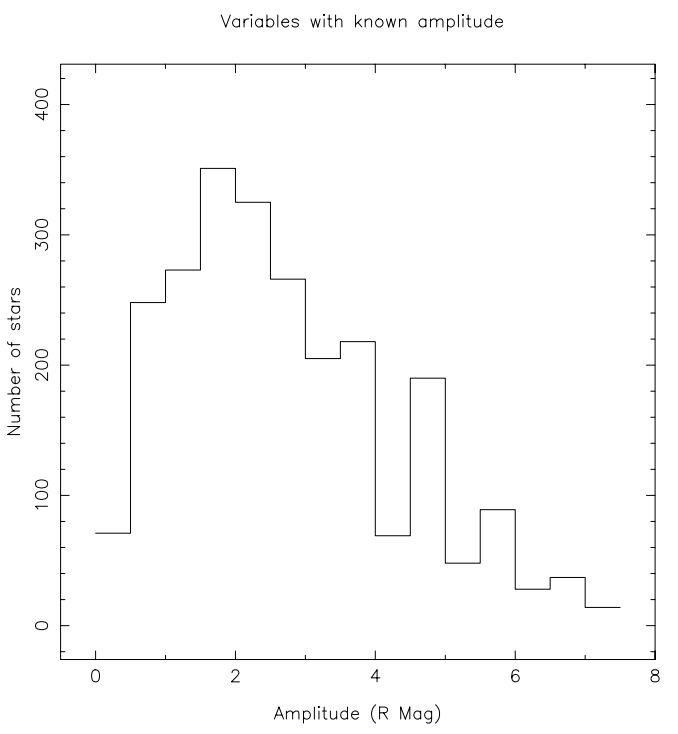

Fig. 4. Histogram of the amplitudes $A$, binned in steps of 0.5 magnitude, for the 4430 variables. Here, stars without known amplitude at minimum light are included in the statistics, with Rmin taken equal to 19 (see text)

stars (Fig. 4). This approximation is believed to be realistic for long period variables (most of the present stars) since:

(i) the magnitude limit achieved on a $30 \mathrm{mn}$ exposure $R$ plate is about 19.5 ;

(ii) the thirteen $R$ magnitudes determined for each star are well distributed over a complete cycle when phased together for typical periods of the detected variables (between 70 and 500 days).

The distributions displayed by Figs. 3 and 4 (in the latter, $50 \%$ of the stars have amplitudes between 1.5 and 3.5 ), are quite similar (the 0.5 magnitude shift observed between the two histograms is not significant). The (ii) statement is supported by a recent study of field O (Alard et al. 1996) showing that 122 of the 150 variables selected for their large amplitude variations $(A \gtrsim 2 \mathrm{mag})$ are Mira type stars, most of them with periods between 150 and 500 days. The analogy shown by the distributions $N / A$ (Fig. 4) and $N /$ Period (Alard et al. 1996) corresponds to the period-amplitude relation existing for this class of stars. We plan to characterize Mira type stars in other parts of the $10^{\circ} \times 10^{\circ}$ field of our programme. These high luminosity objects show a definite period-luminosity relation and thus are good distance indicators. Infrared $J$ and $K$ photometry of these stars would then be worthwhile in order to evaluate the variation of the interstellar extinction with the distance modulus in the direction of the galactic centre.

Acknowledgements. We would like to thank all the members of the MAMA team for assistance during the scanning of the photographic material. We are indepted to Gerard Marichy and Daniel Robert for their technical help.

\section{References}

Acker A., Cuisinier F., Stenholm B., Terzan A., 1992, A\&A 264,217

Alard C., Terzan A., Guibert J., 1996, A\&AS 120, 275

Cuisinier F., Terzan A., Acker A., 1993, A\&A 277, 203

Cuisinier F., Terzan A., Acker A., 1994, A\&A 287, 227

Kholopov P.N., et al., 1985, 1987, General Catalogue of Variable Stars, Nauka, Moscow, Vols. II and III (GCVS)

Robichon N., Turon C., Makarov V., et al., 1995, A\&A 304, 132

Röser S., Bastian U., 1991, PPM Star Catalogue, Vol. III, Publications A.R.I., Spektrum

Röser S., Bastian U., 1993, PPM Star Catalogue, Vol. IV, Publications A.R.I., Spektrum

Terzan A., 1965, C. R. Acad. Sc. Paris 291, 3974

Terzan A., 1990, The Messenger 59, 41

Terzan A., Bernard A., 1981, A\&AS 46, 49

Terzan A., Bijaoui A., Ju K.H., Ounnas C., 1982, A\&AS 49, 715

Terzan A., Chatagnat M., Dubet D., 1978, J. Opt. 9, 121

Terzan A., Gosset E., 1991, A\&AS 90, 451

Terzan A., Ounnas C., 1988, A\&AS 76, 205 


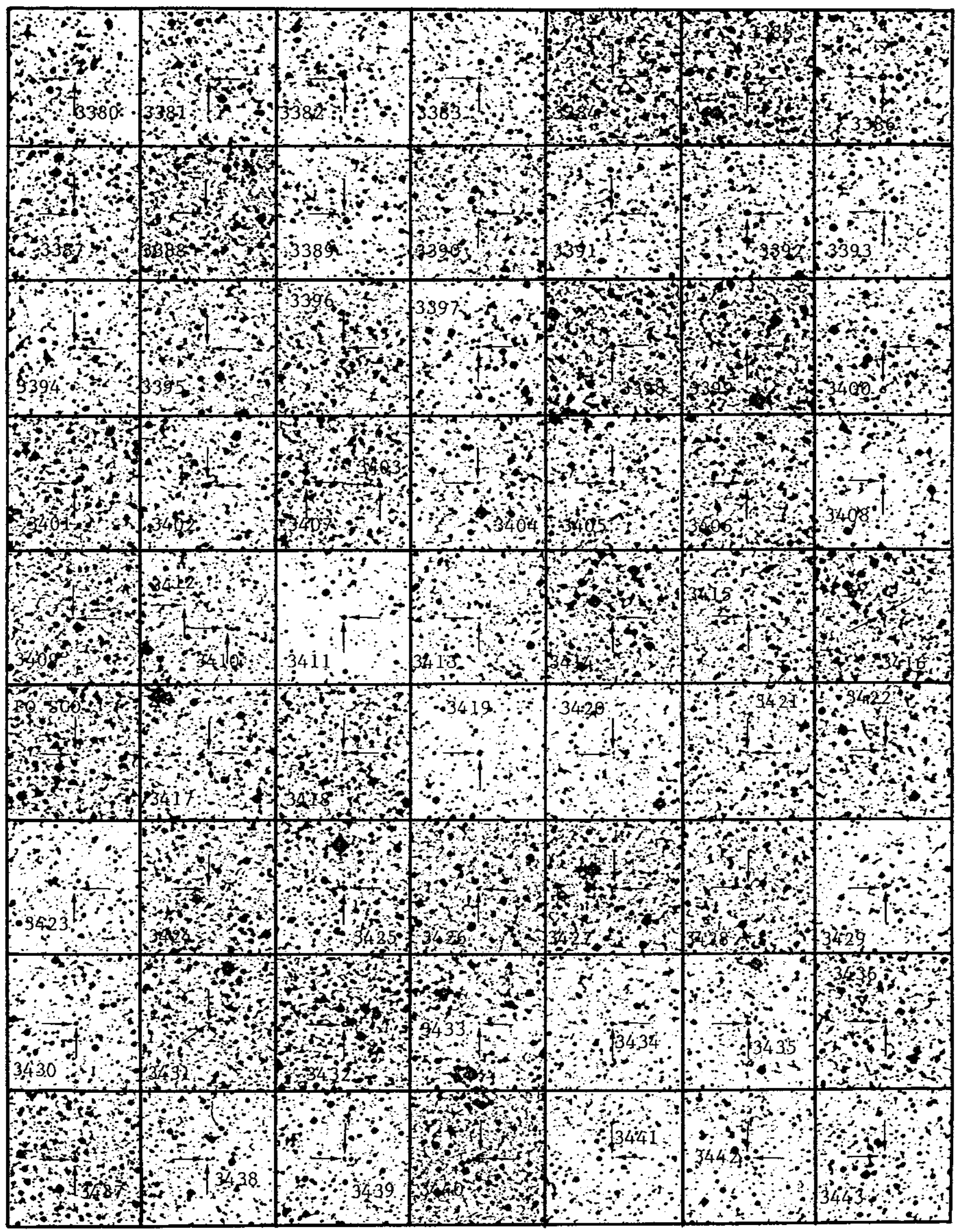

Plate 1 


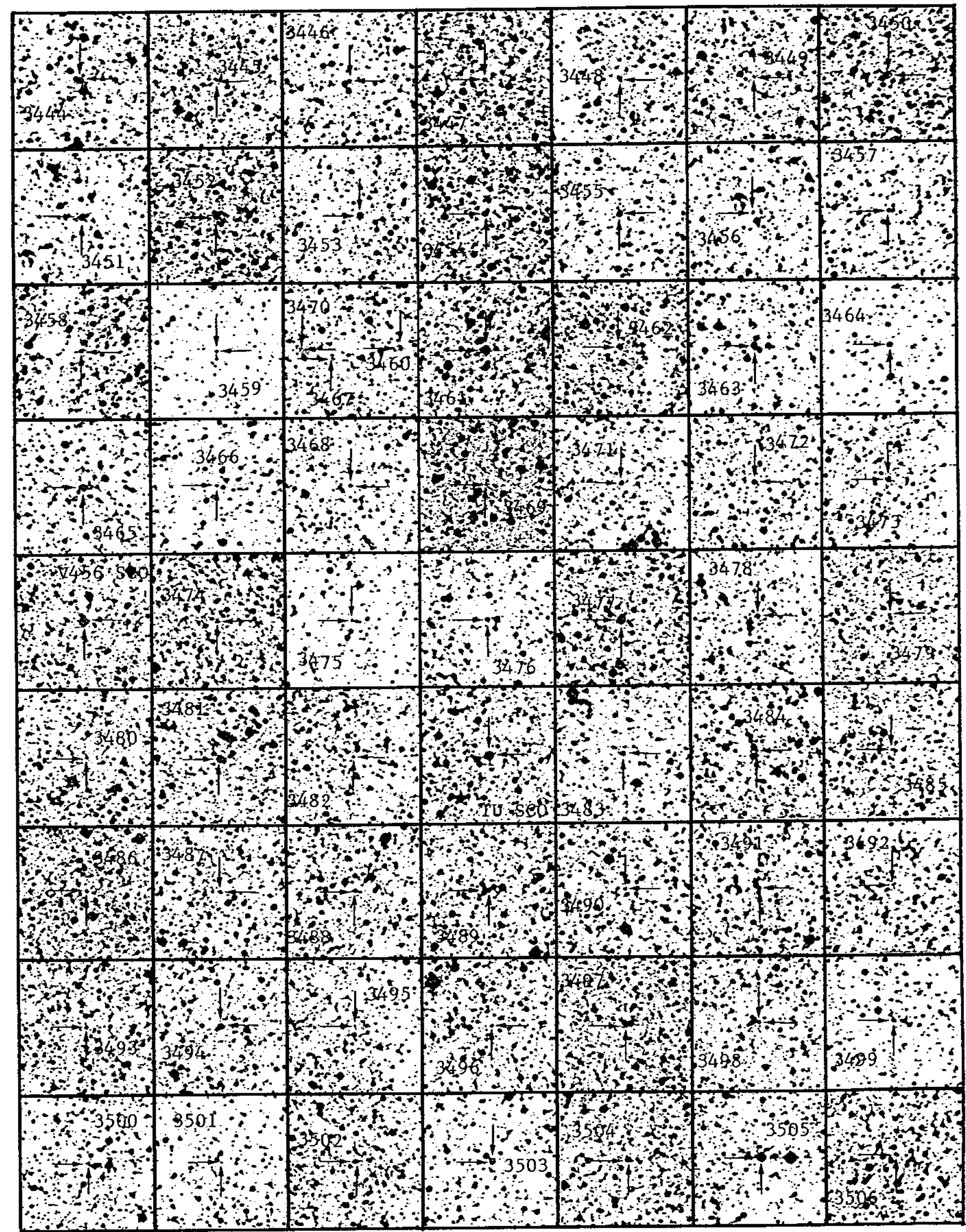

Plate 2 


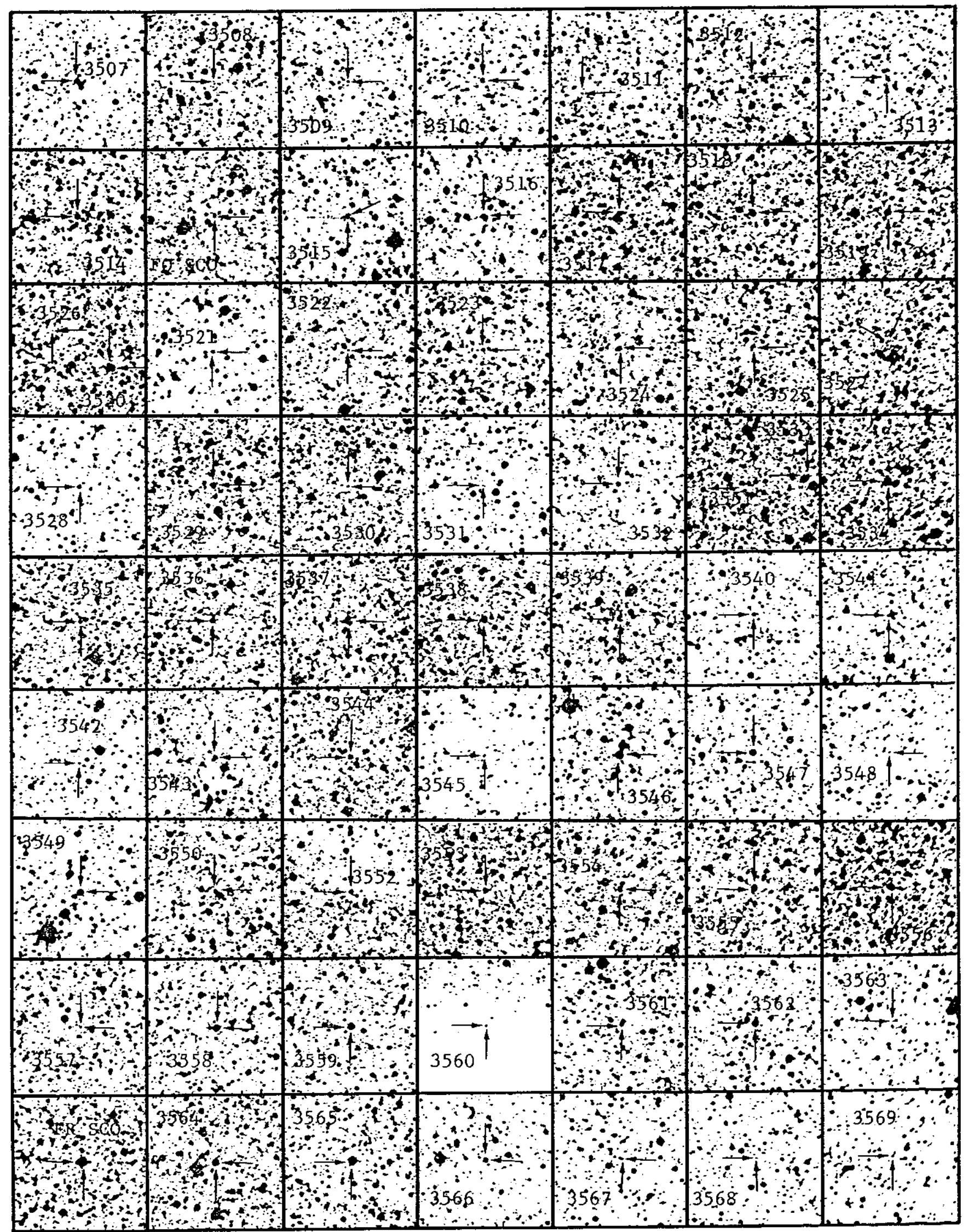

Plate 3 




Plate 4 


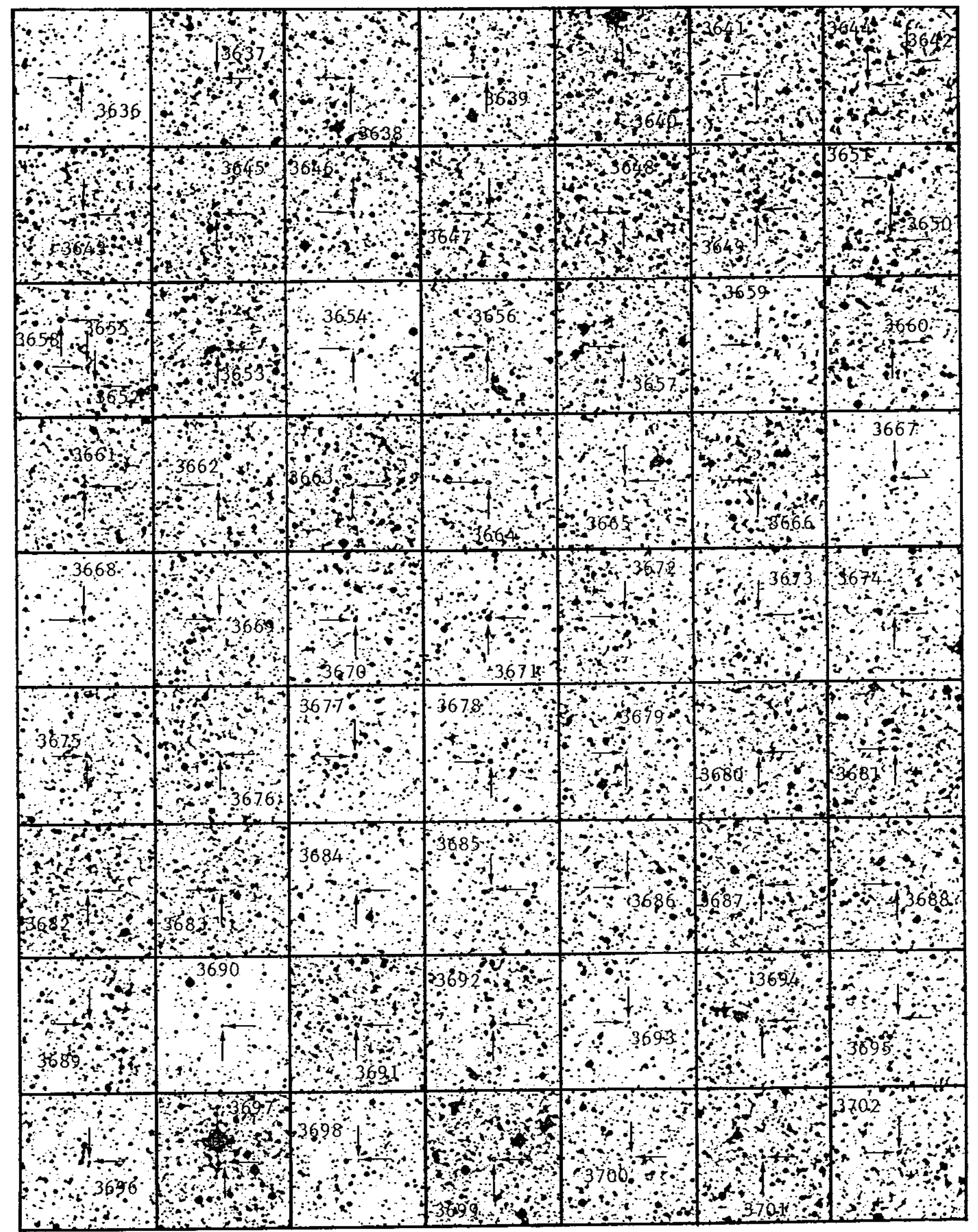

Plate 5 


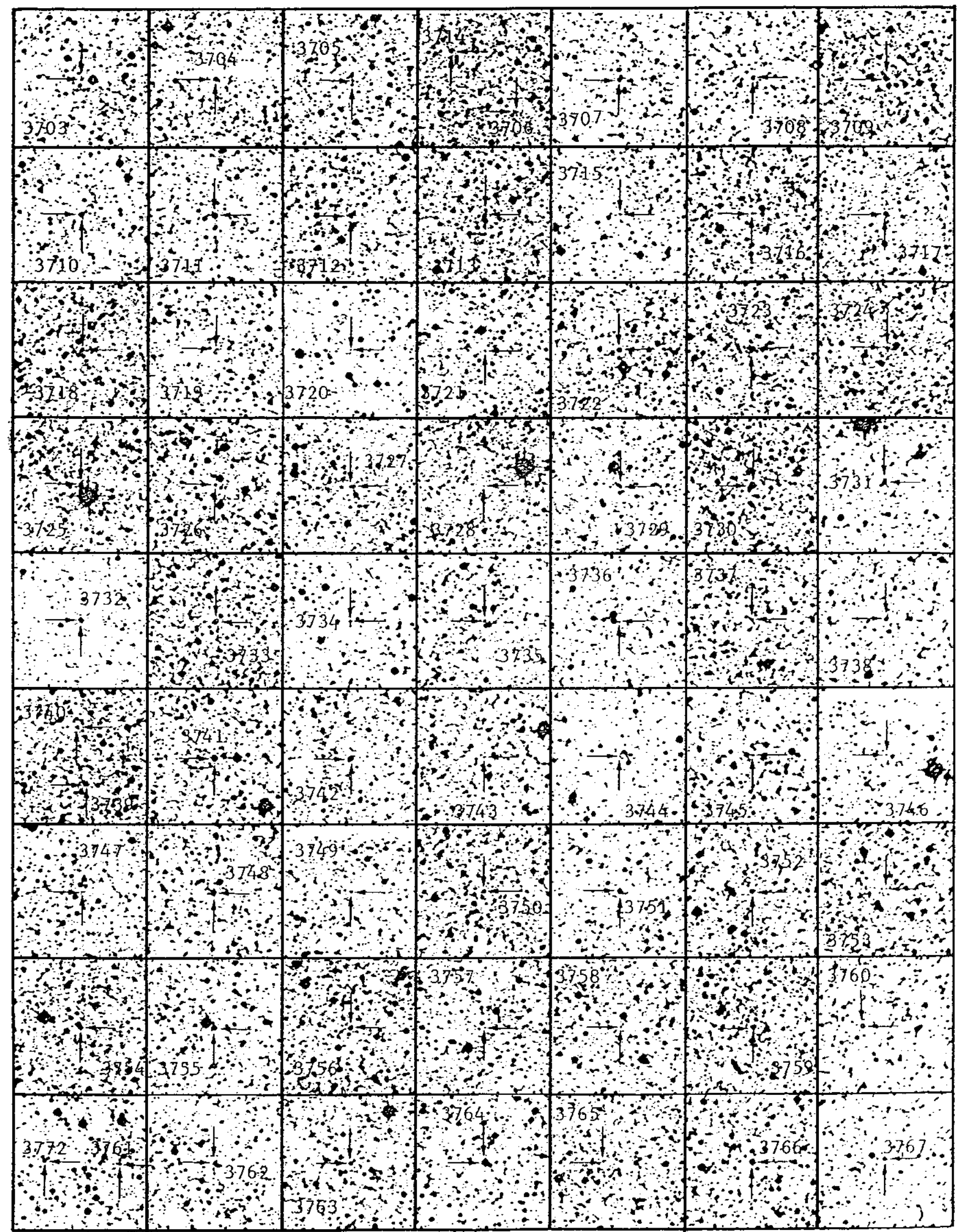

Plate 6 


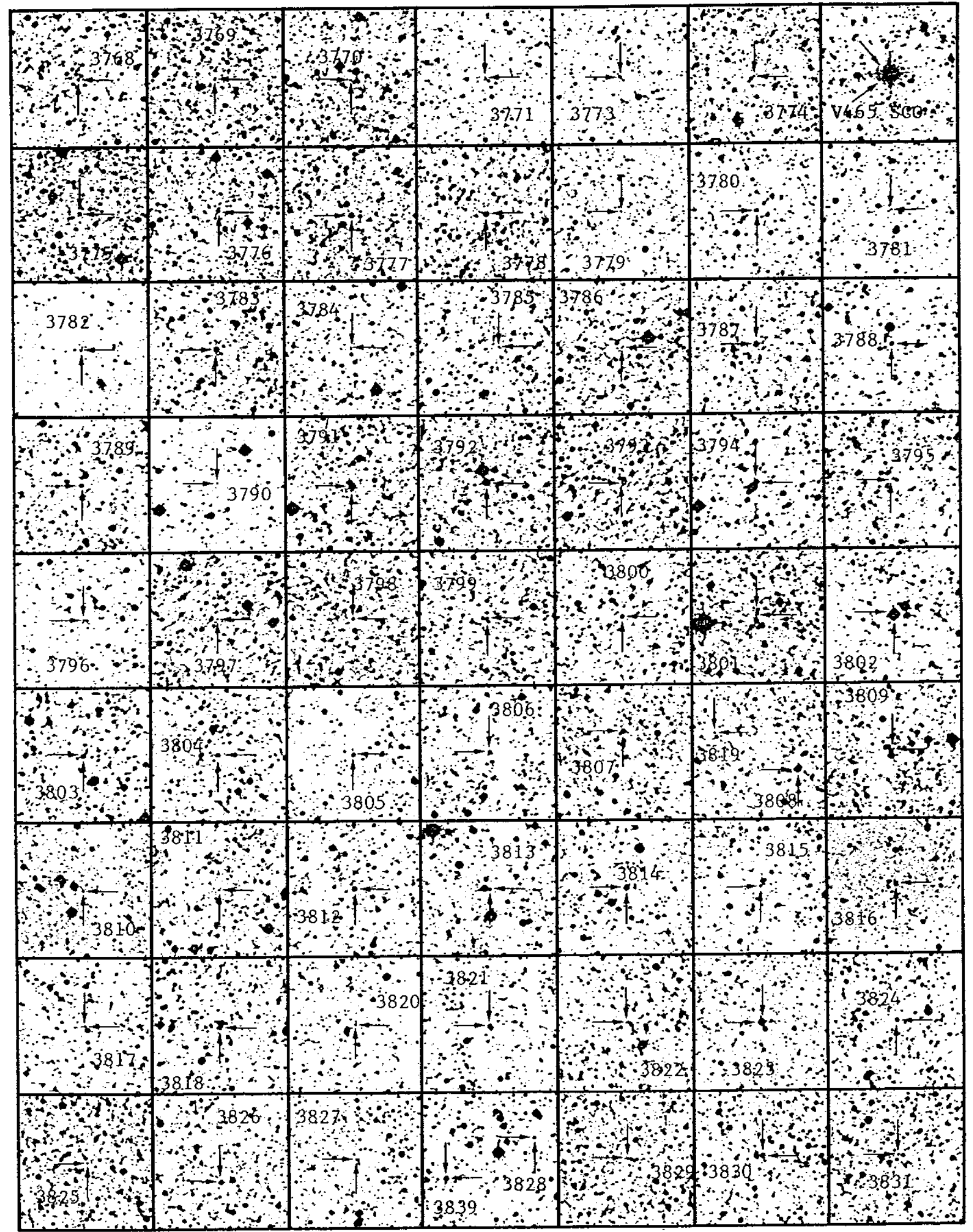

Plate 7 


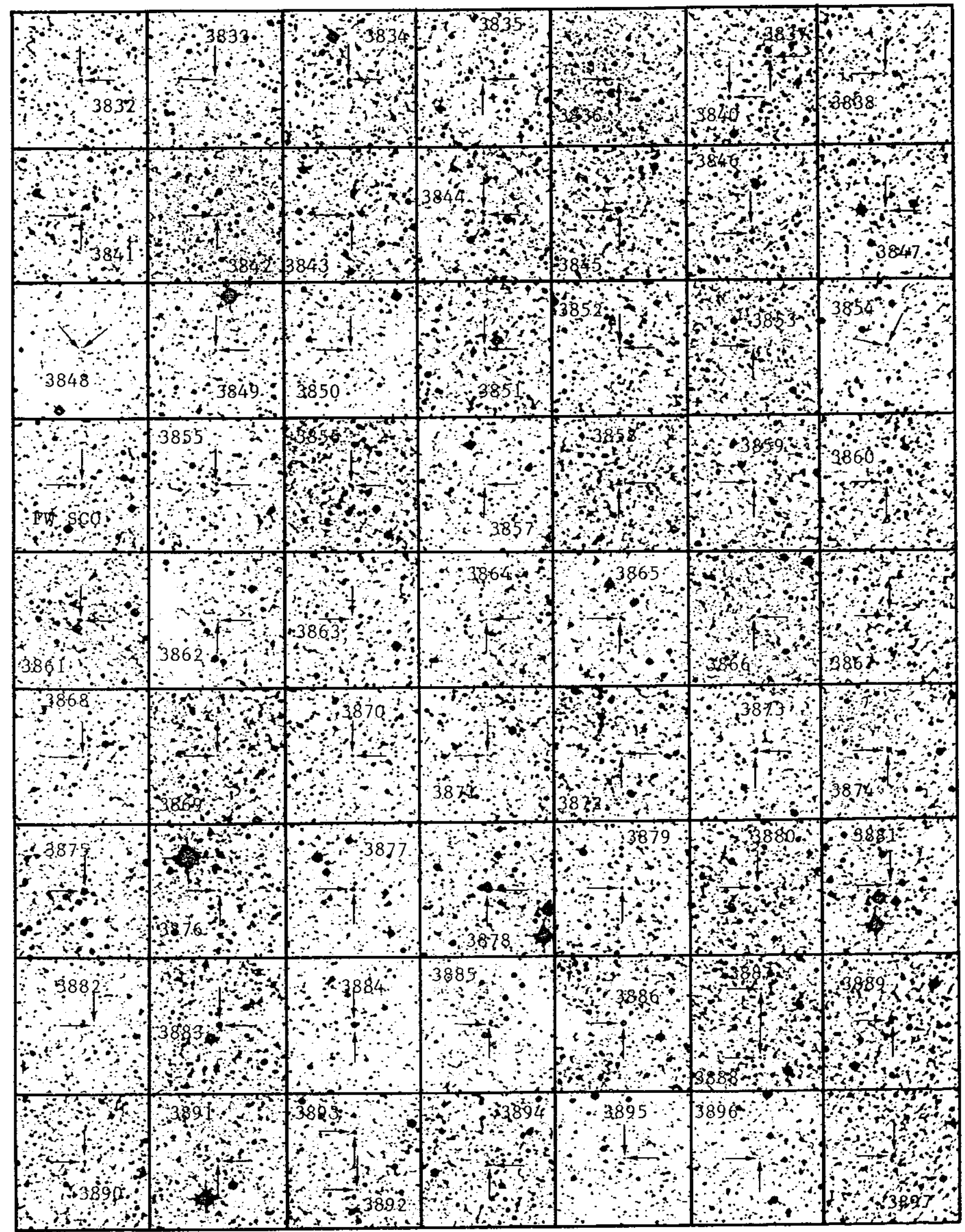

Plate 8 


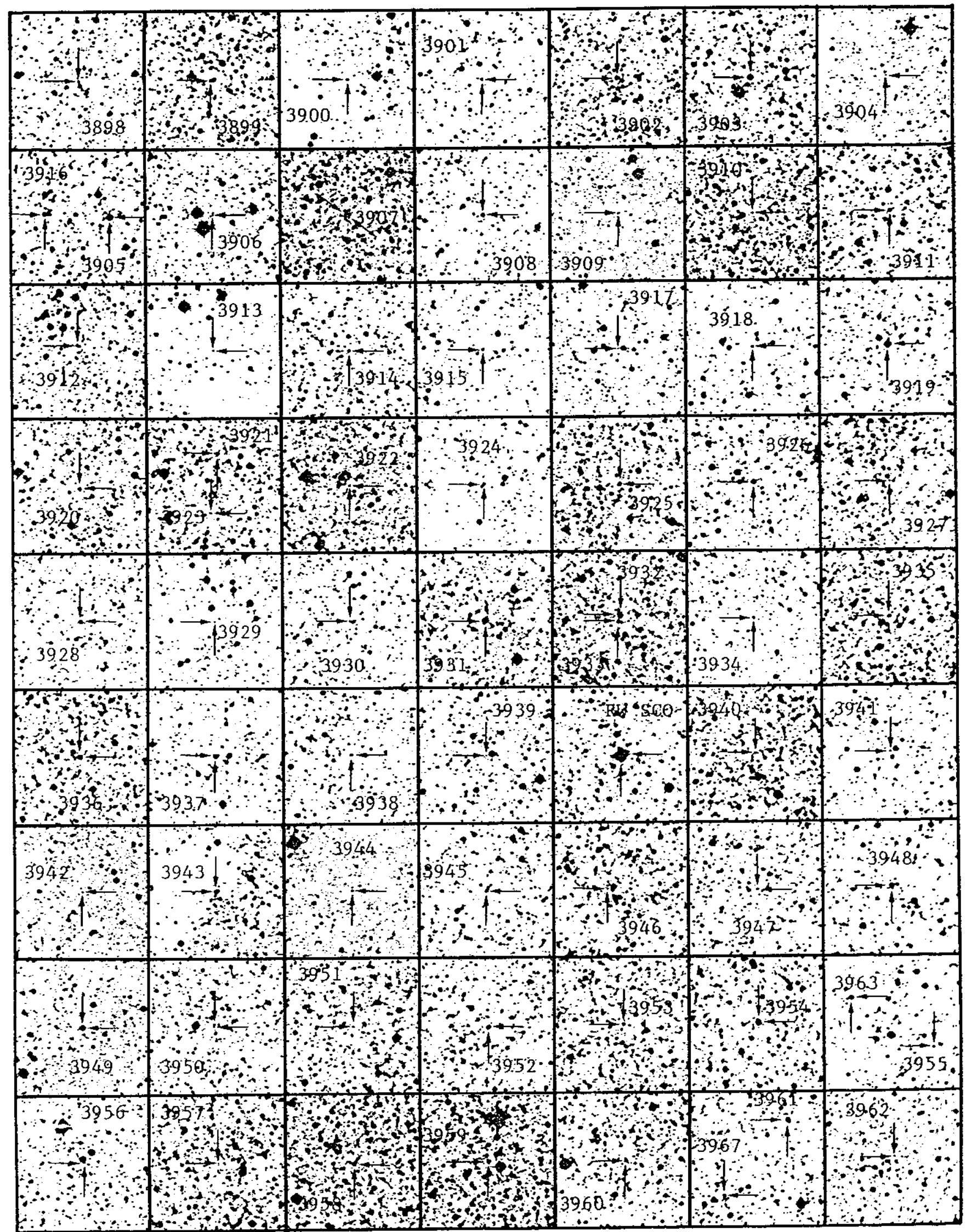

Plate 9 


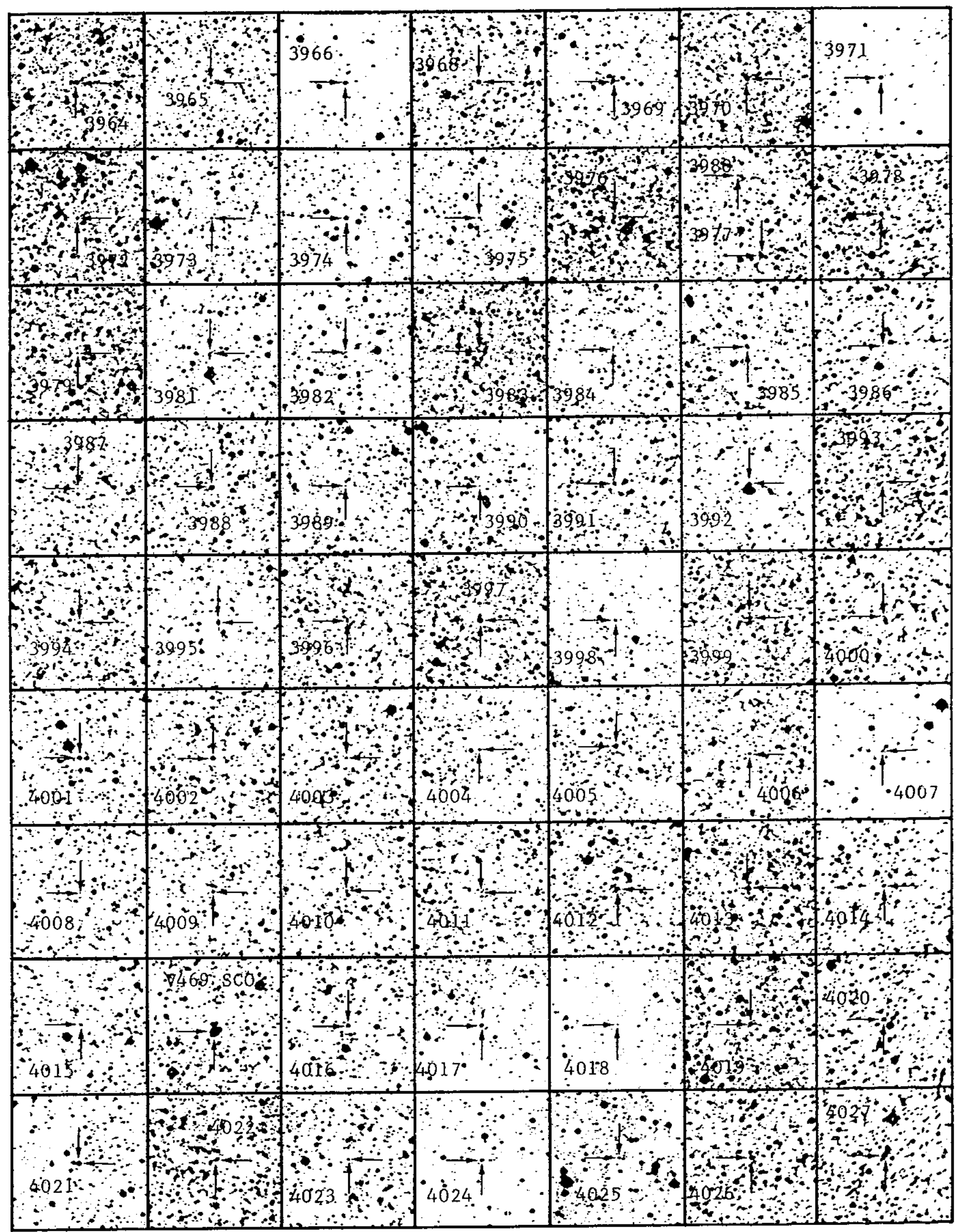

Plate 10 


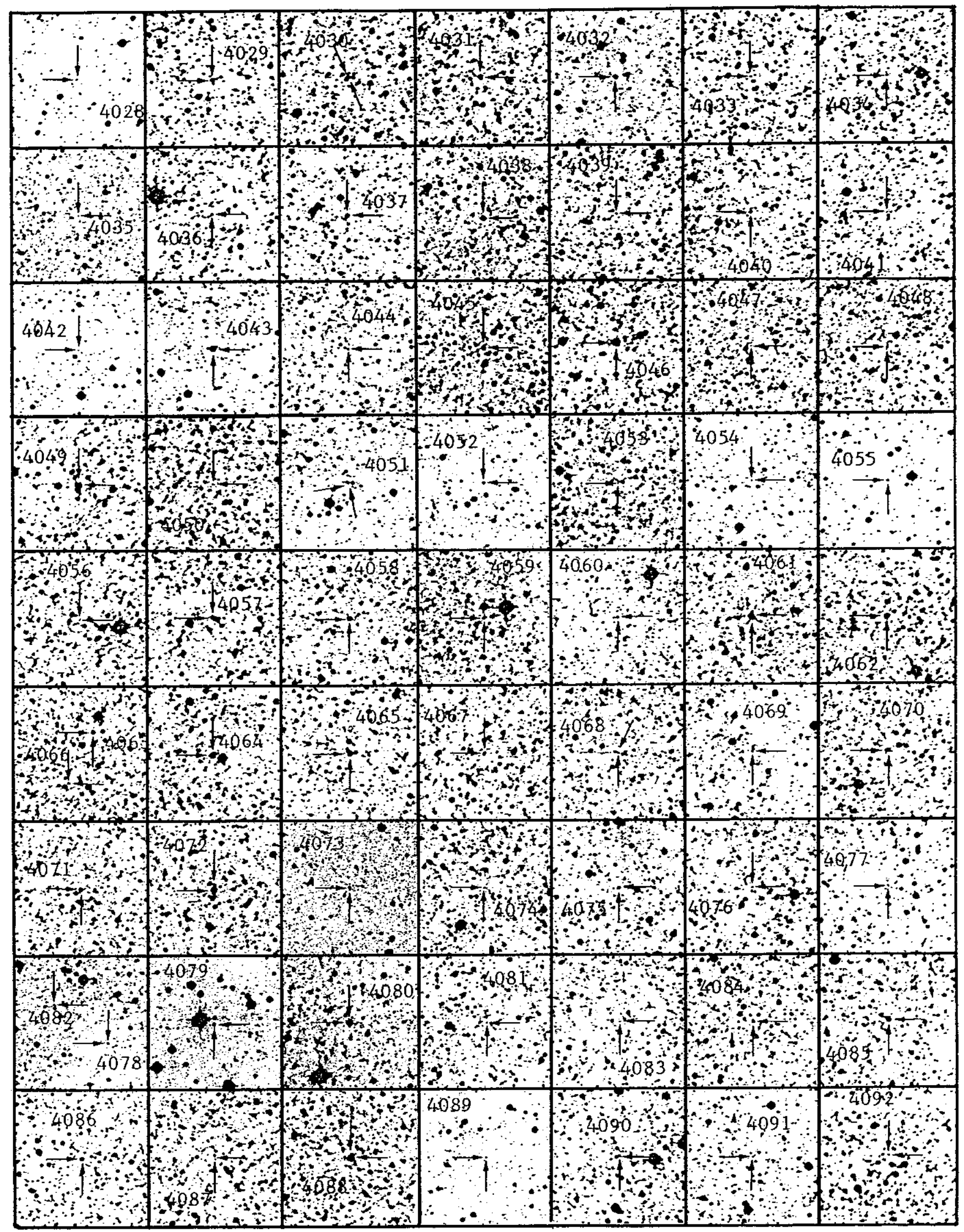

Plate 11 


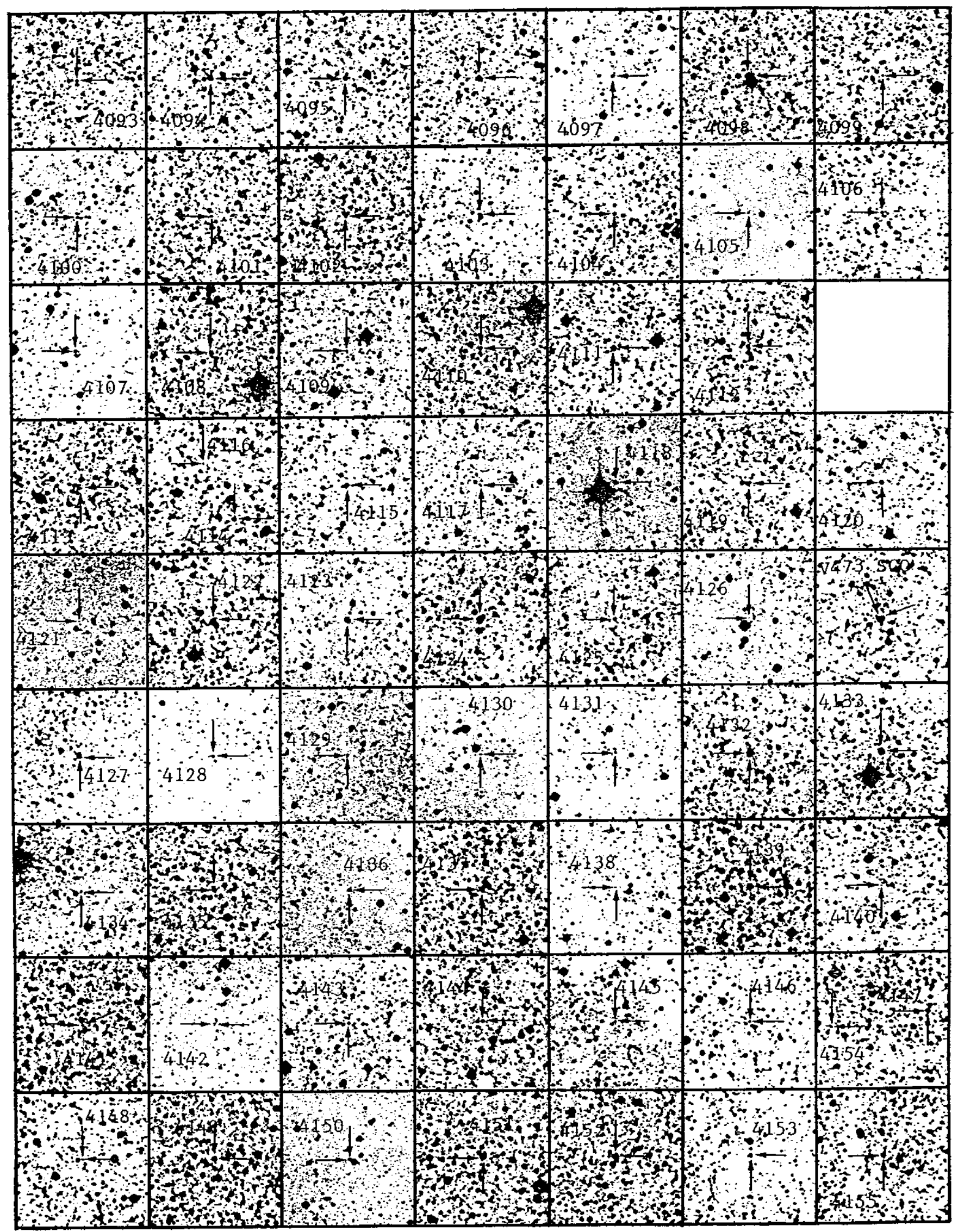

Plate 12 


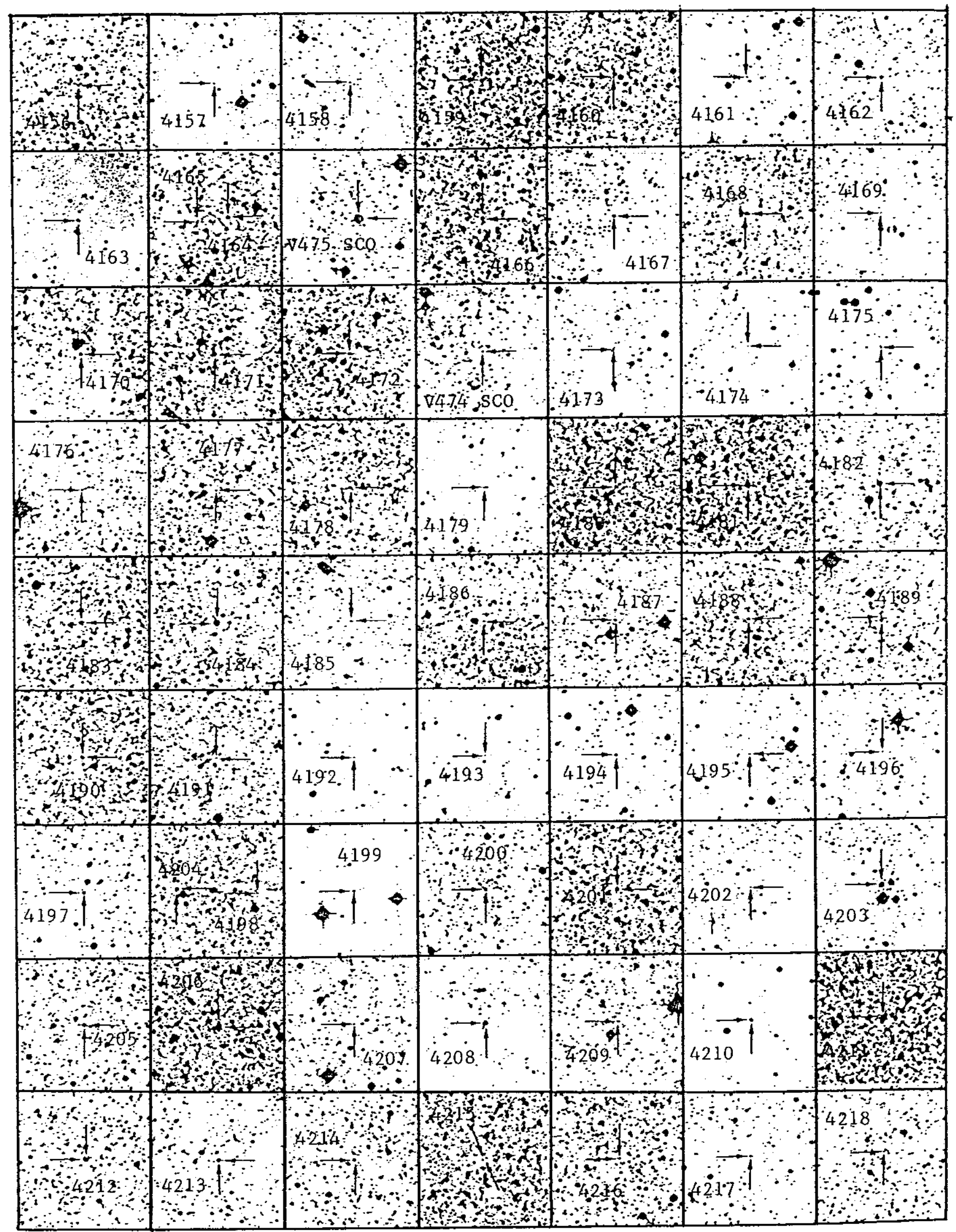

Plate 13 


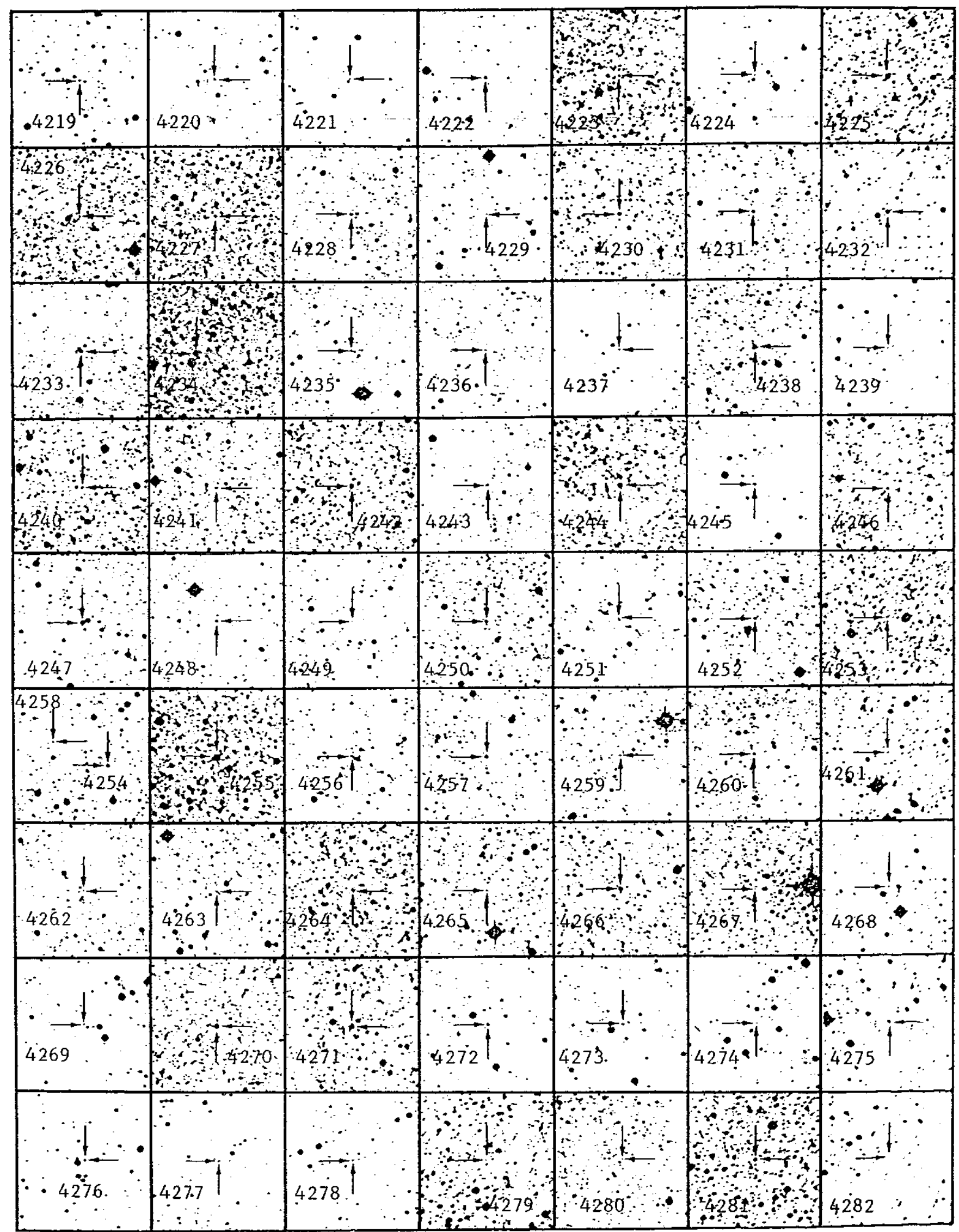

Plate 14 


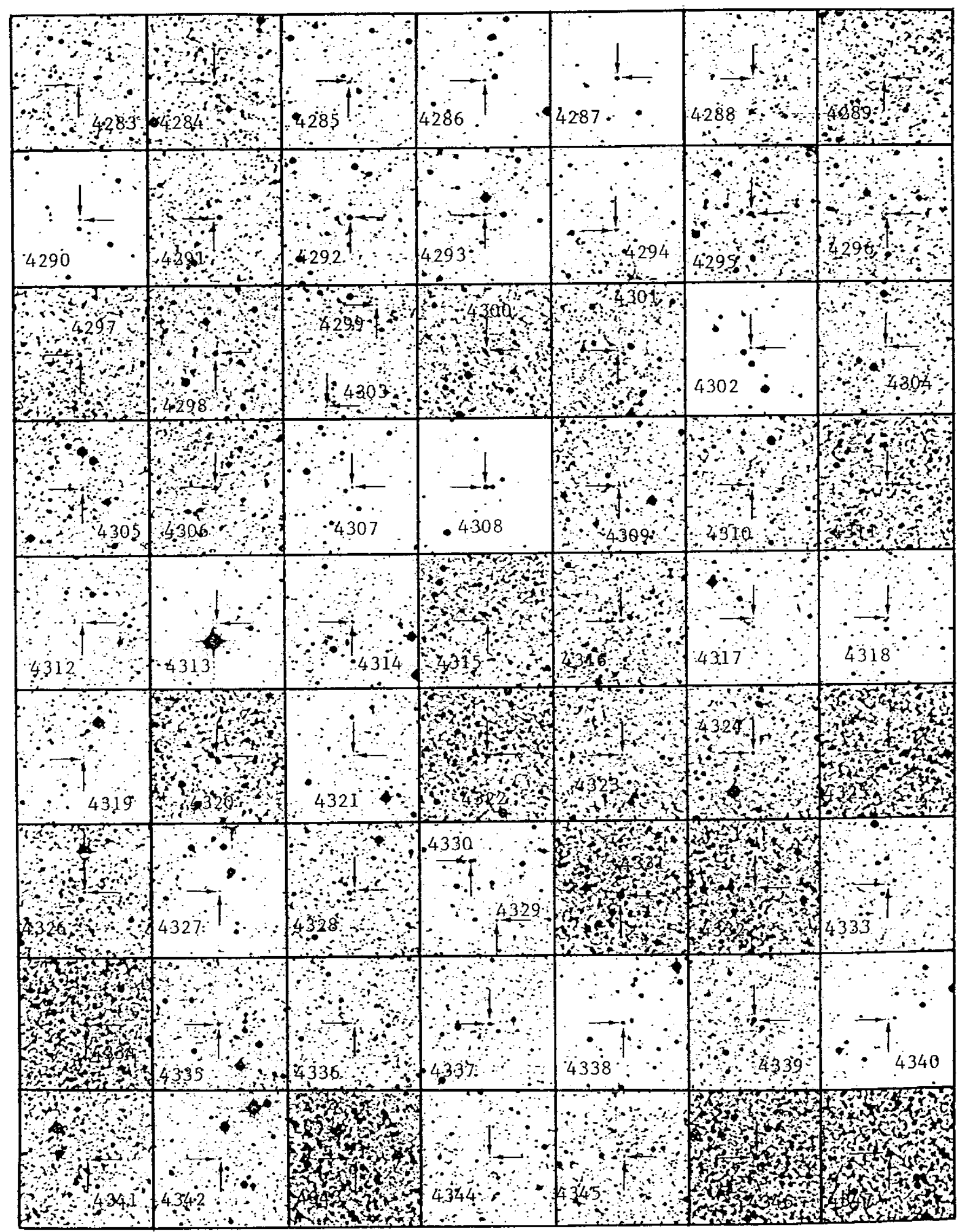

Plate 15 


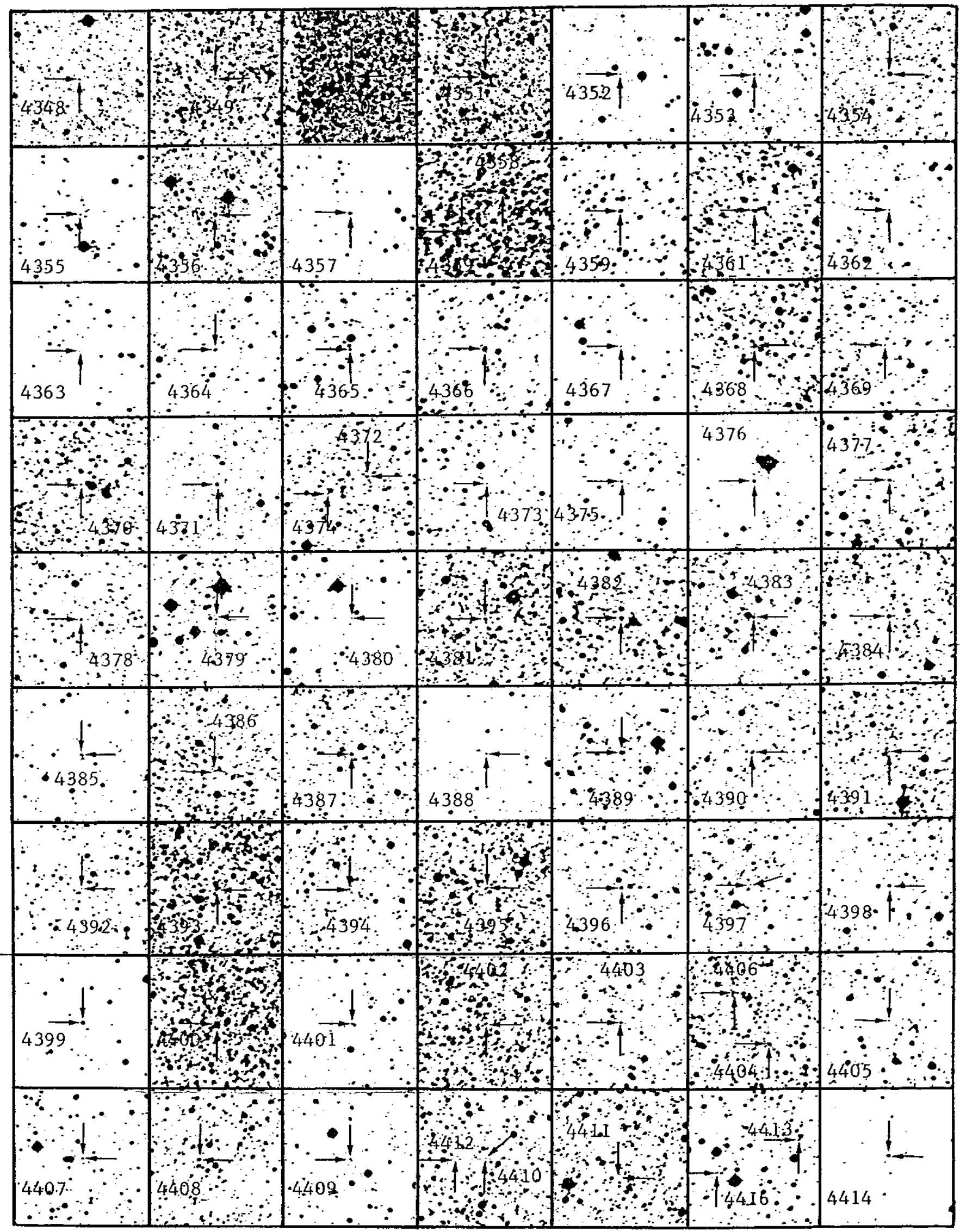

Plate 16 


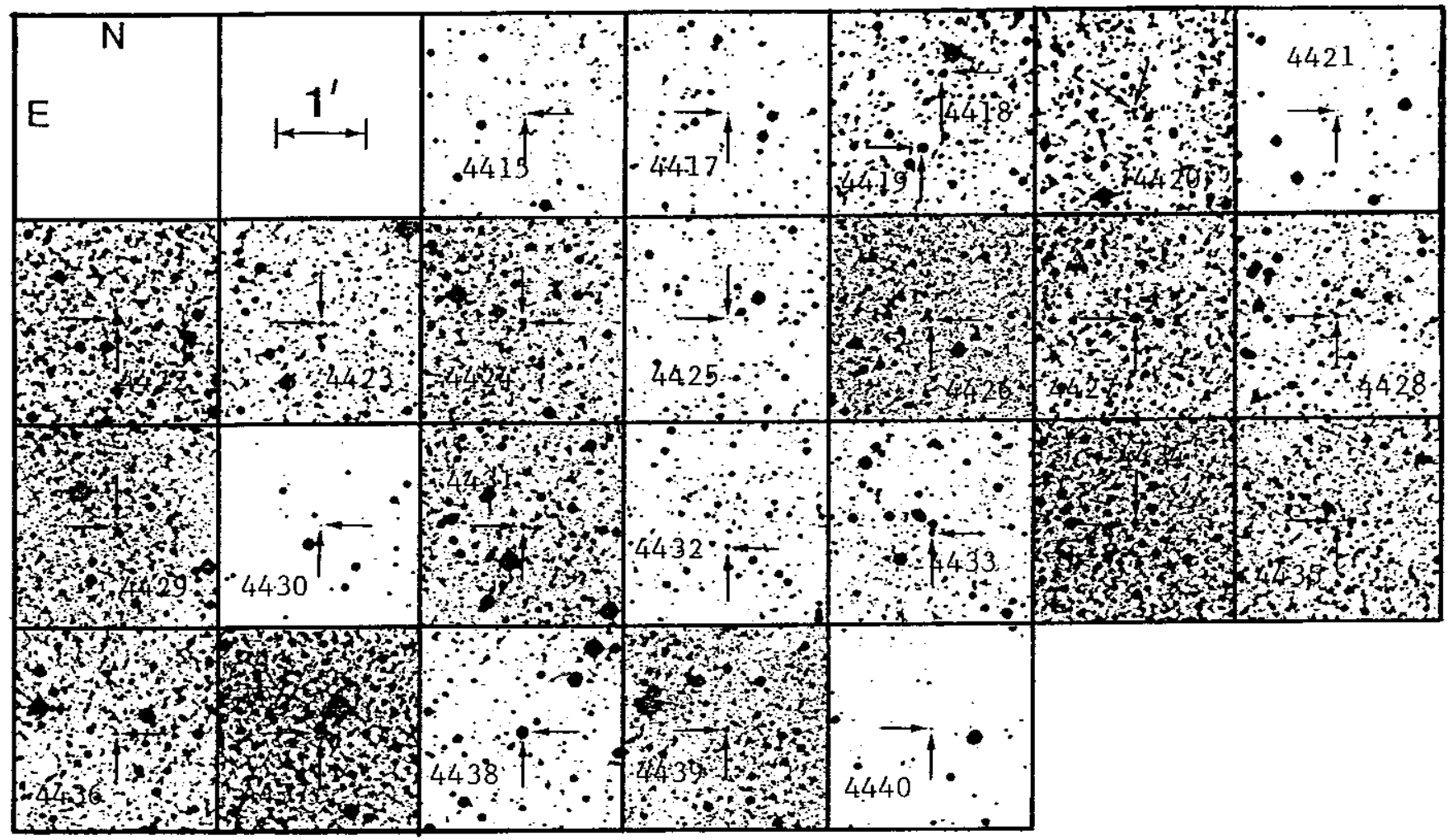

Plate 17 